\title{
An Efficient Noise Separation Technique for Removal of Gaussian and Mixed Noises in Monochrome and Color Images
}

\author{
Satish Kumar Satti1, Suganya Devi K, Prasenjit Dhar, P Srinivasan
}

\begin{abstract}
Images are often affected by different kinds of noise while acquiring, storing and transmitting it. Even the datasets gathered by the various image acquiring devices would be contaminated by noise. Hence, there is a need for noise reduction in the image, often called Image De-noising and thereby it becomes the significant concerns and fundamental step in the area of image processing. During image de-noising, the big challenge before the researchers is removing noise from the original image in such a way that most significant

properties like edges, lines, etc., of the image, should be preserved. There were various published algorithms and techniques to de-noise the image and every single approach has its own limitations, benefits, and assumptions. This paper reviews the noise models and presents a comparative analysis of various de-noising filters that works for color images with single and mixed noises. It also suggests the best filter for color that involve in producing a high-quality color image. The metrics like PSNR, Entropy, SSIM, MSE, FSIM, and EPI are considered as image quality assessment metrics.
\end{abstract}

Keywords - De-noising Edge preserving filtering $\bullet$ Spatial Domain Filters • Transform Domain Filters • Non Local Means • DnCNN • Gaussian noise • Mixed Noise $\bullet$ PSNR • MSE • EPI • FSIM $\bullet$ SSIM.

\section{INTRODUCTION}

While the image acquisition and image transmission process, digital images can be corrupted by noisy pixels, thereby degrading the image quality. Noise in the image may be caused by sensors, scanners, optic defects, relative motion, and environmental conditions. In the area of image processing and computer vision the de-noising, an image is one of the basic challenges, where the first underlying goal is to suppress the noisy pixels from the image with preserving geometric and structural properties of the image, such as sharp structures, corners, and lines to achieve high visual quality.

Second, filtering of homogeneous regions to get improvement in the SNR (Signal to Noise Ratio). In image registration and image enhancement, de-noising an image serves as a pre-processing and post-processing technique. The major applications of image de-noising find in remote sensing, medical images, surveillance, etc. Image de-noising play a vital role in medical imaging to investigate the valid diagnosis and thereby helping doctors to do adequate therapy. Different types of noise [1] are presently based on

\footnotetext{
Revised Manuscript Received on July 18, 2019.

Satish Kumar Satti1, Research Scholar NIT Silchar-Assam. (sskumar789@gmail.com)

Dr Suganya Devi K, Assistant Professor NIT Silchar-Assam. (suganyanits@gmail.com)

Prasenjit Dhar, Research Scholar NIT Silchar-Assam. (prasenjitdhar.cse@gmail.com)

Dr P Srinivasan, Assistant professor NIT Silchar-Assam. (srininits@gmail.com)
}

the type of modality used. Most Natural images, SAR images, Underwater images, rock images are degraded with additive random noise and it is modeled as a Gaussian distribution, Noise in Magnetic Resonance Image (MRI) can be modeled asRician [2] distribution, speckle [3] noise in Ultrasound (US) images are modeled as Raleigh distribution [4], Photon noise in Positron Emission Tomography (PET) images can be modeled as Poisson distribution. There are many approaches to de-noising the image $[5,6]$ Spatial domain filters, Transform domain filters, and neural network-based filters. The Spatial domain filters are welldefined in the 2D image plane. In this, de-noising filters are directly operated on pixel intensities. These are used to remove the noise using the weighted averaging process. Spatial filtering [7] can be classified into two categories: 1. Linear 2. Nonlinear. The linear filtering in which an output pixel value is a linear weighted averaging of pixel intensities in the input pixel's neighborhood. Some of the linear filters are a mean filter, Weiner filter, Gaussian filter. The operation performed on the pixels within the filter mask is non-linear. Some of the nonlinear filters [8] are Median filters, Total variance, Non-local mean, bilateral, max filter, min filter. In transform domain filtering methods noisy image is converted into some transform and then de-noising filters are enforced to the transformed image. The Transform domain filters divided into Curvelet, Nonlocal based (BM3D, BM 4D) and Contourlet, [9], Data adaptive and Non-data Adaptive filters (Wavelet Domain). Wavelet filters are used to suppress noise from the image by preserving the basic structures of the original image. The popular Wavelet domain filters are SURE, Bayes, Bivariate, Non-local mean, shrinkage rules-based filters.

To optimize and automate the process of suppressing the noise from image novel filters called Neural Network (NN) filters are used. Some types of Neural Network-based filters are Cellular based filters, Auto encoder based filters, and Convolutional based filters.

\section{NOISE MODELS:}

During the image acquisition process, due to faulty in device hardware, camera sensor or due to poor environmental conditions images are affected with distinct classes of noises namely Gaussian, Impulse, Speckle, and Poisson. Probability Density Function (PDF) or Histogram techniques are chosen to model and illustrate these noise models.

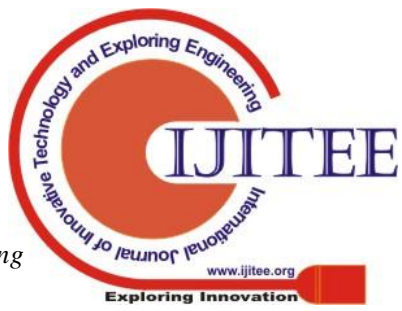




\section{AN EFFICIENT NOISE SEPARATION TECHNIQUE FOR REMOVAL OF GAUSSIAN AND MIXED NOISES IN MONOCHROME AND COLOR IMAGES}

\subsection{Gaussian Noise (GN):}

The Gaussian noise is caused by low light or high temperature and it is getting added to the image at the time of capturing the image. Gaussian Noise follows Gaussian distribution. GN corrupts each gray pixel values in the image and its strength is computed with its standard deviation and mean. An original image and an image deprived by Gaussian are shown in Fig1.
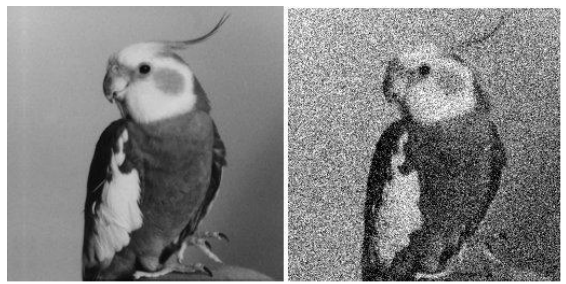

Fig. 1 (a) Original Image. b) Gaussian Noise Image.

\subsection{Impulse Noise:}

Impulse Noise (IN) is the noise produced during image capturing due to defected camera sensors, malfunctioning of the device's hardware, transmission errors or memory errors. It is classified into two types i) Salt and Pepper and ii) Random Valued noise. i) Salt and pepper noise is dark pixel values in bright regions and bright pixel values in dark regions. In this, the grey level value 255 is considered as salt (white) and 0 is considered as pepper (black) and it seems like black and white dots on an image. ii) Random valued noise takes a gray level values from 0 to 255 . An input image and an image deprived by impulse are shown in Fig2.

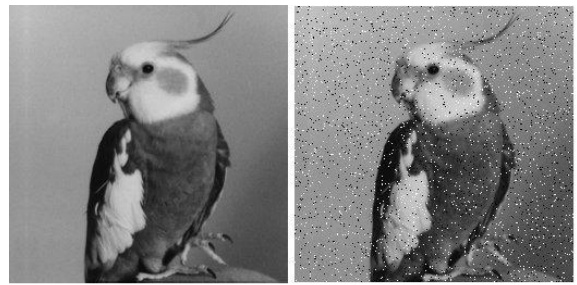

Fig. 2 (a)Original Image. (b) Impulse Noise Image.

\subsection{Speckle Noise:}

It is a multiplicative noise introduced in medical ultrasound (US) images, radar images, optical coherent tomography, and Synthetic Aperture Radar (SAR) images. Speckle noise degrades the contrast and resolution of an image resulting in degrading the quality of an image. Its PDF follows gamma distribution". Fig 3 An original image and an image deprived by speckle.

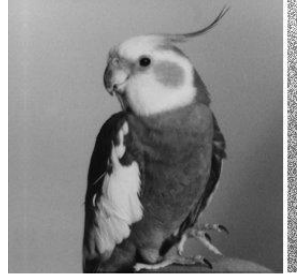

Fig. 3 (a) Original Image.

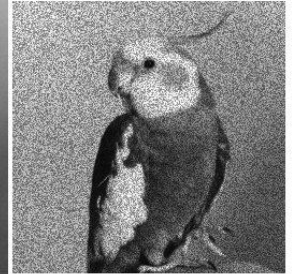

(b) Speckle Noise Image. produced by analog to digitally converted errors resulting in

\subsection{Poisson Noise:}

It is also called as Photon noise and follows the Poisson distribution. It occurred when the amount of photons detected with an image sensor is not adequate to deliver visible statistical information. Fig 4 shows the image with impulse noise.
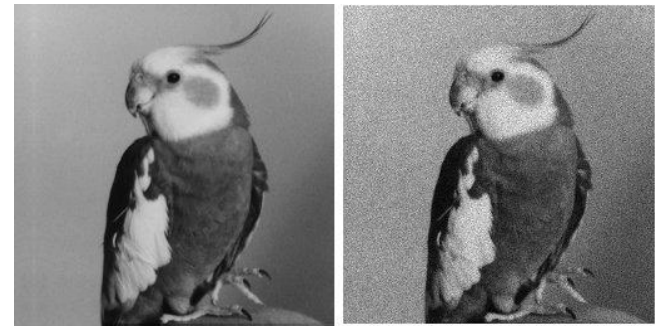

Fig. 4 (a) Original Image.(b) Poisson Noise Image.

\section{DE-NOISING FILTERS:}

Filters play a vital role in de-noising an image. It is a methodology to improve image quality. There are two basic image de-noising techniques focusing on preserving edge and fine structure of an image: i) Spatial domain filters and ii) Transform domain filters.

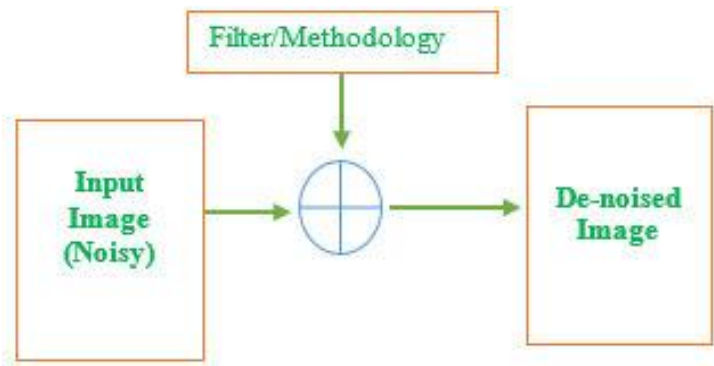

Fig. 5 Image De-noising Process

\subsection{Spatial Domain Filtering:}

It is considered as an image processing technique where each pixel is measured by its intensity and the spatial coordinates. It can manipulate the pixel attributes i.e., manipulation of intensity, manipulation of spatial location, manipulation of both intensity and location. Spatial domain filters are categorized into linear and nonlinear filters.

\subsubsection{Linear filters:}

The linear filters like Mean, Gaussian, and Wiener are mainly used to reduce the Additive White Gaussian Noise (AWGN)

\section{(a) Mean Filter:}

It smoothens the image by reducing the variation in terms of intensity between adjacent pixels. In this, the center pixel value is replaced with averaging all the neighborhood pixels. It is instigated with a convolution mask and delivers a weighted summation of pixels and its neighbor pixels as an outcome. The mask or kernel is a square matrix and generally, a 33 square matrices are considered. The mask or kernel is a square matrix and frequently a 33 square matrices 
are considered. It returns dark if the summation of the coefficients of kernel equals to zero. If the summation of coefficients equal to one, then no change in the average brightness of the image. Licheng Liu et al [10] introduced a new filter called Weighted Mean Filter (WMF) to remove Impulse Noise in an image. To de-noise the impulse noise, Liu et al presented a 2-stage noise detector. First, to detect the noise candidates, a Rank Ordered Difference of RankOrdered Absolute Difference (ROD-ROAD) scheme is applied. Second, applied Minimum Edge Pixels Difference (MEPD) to find edge pixels from noisy candidates. Finally, an interactive de-noising algorithm is developed by incorporating a two-stage image noise detector and a new WM filter. Cengizkandemir et al [11], UWMF (Unbiased Weighted Mean Filter) is developed to de-noise high-density impulse. UWMF abolishes spatial bias produced through the asymmetric delivery of degraded pixels by recalibrating the weights of uncorrupted pixels and the resulted intensity value is exchanged with the weighted mean by using the recalibrated weights.

\section{b.Gaussian Filter:}

The Gaussian noise is caused by low light or high temperature and it is getting added to the image at the time of acquiring the image. This type of noise increases random Gaussian distributed noise to an original pixel value. To suppress such type of noise from images, Gaussian filter [12] is used. Vishal Choudhry et al [13] suggested a new methodology by amalgamating recursive and Gaussian filter to split source image into constant, high varying and low varying feature images. The merit of this filter combination is clean removal of features. Gaussian filters are used to suppress the noise but it removes the fine details of an image. G Deng et al [14], proposed an Improved Gaussian filter to de-noise and detecting edges in an image.

\section{c. Wiener Filter:}

To reduce additive noise in the image, a spatial filter called wiener filter is developed by Norbert Wiener. Wiener filter is worked on the least squared principle to diminish the Mean Squared Error (MSE) between real output and anticipated output. Fourier transforms techniques and means squared techniques are used to implement the Wiener filter. In Fourier transform technique priory knowledge of the original image is required to de-noise the image whereas no such prior knowledge is required for mean squared technique. Aziz Makandar et al [15], suggested that as the mammography images are noisy, low contrast, blur and fuzzy it is required to enhance the mammography images with Wiener filter to bring out specific features like mass and microcalcification and to highlight certain characteristics for early detection and easy diagnosis of breast cancer. So, from the above discussion, it is clear that linear filters distort the sharp edges, eradicate lines and additional fine proprieties of an image.

\subsubsection{Non-Linear Filters:}

These filters used to de-noise the image without misplacing the significant features like edges, lines of an image.

\section{i. Median Filter:}

These are applied to suppress the noise from an image with retaining edges and basic fine details of the image. It is robust when compared to the mean filter. In this technique, the center pixel of a window is supplanted with a median value of neighboring pixels of the center pixel value in a window. First, sort the neighboring pixel values of the center pixel in either ascending order or descending order then compute median and replace it with center pixel value. Example: Consider 3 x 3 square matrix form below pixel values Consider pixel value at $(3,3)$ i.e.142. Adjacent of this pixels are 17, 152, 110, 134, 142, 109, 154, 130 and 136.Now sort it in ascending order then we will get 109 , $110,117,130,134,136,142,152,154$ and median among these is 134. Now replaceall intensity values with the median value 134 including the center pixel value 142 . To suppress the random-valued impulse noise a two-phase median filter is proposed by Jianjun Zhang [16]. First, corrupted noisy pixels are identified by Additive center weighted median filter. Second, a Median Filter based interactive method is used to restore noisy candidates with preserving edges and noise-free pixels. Jiansheng Chen et al [17], suggested that automation of extracting features from the image a median filter is incorporated in Convolutional Neural Networks (CNNs). The filter layer is the first layer in the $\mathrm{CNN}$ framework that receives an image as the input and produces Median Filtering Residual (MFR) as output. Then, we acquire multiple features for further classification by the way of alternating convolutional layers and pooling layers to learn hierarchical representations.

\begin{tabular}{|l|l|l|l|l|}
\hline 127 & 149 & 177 & 113 & 152 \\
\hline 122 & 117 & 152 & 110 & 120 \\
\hline 124 & 134 & 142 & 109 & 114 \\
\hline 114 & 154 & 130 & 136 & 114 \\
\hline 136 & 157 & 157 & 200 & 147 \\
\hline
\end{tabular}

Fig. 6 calculating the median value of a pixel neighborhood

\section{ii.Anisotropic Diffusion Filter $(A D)$ :}

De-noising an image without losing the basic features like edges, corners, and sharp structures is a thought-provoking task. Anisotropic Diffusion (AD) filter [18] suppresses the noise from an image with preserving the basic structures of the image. To save the textures in image AD filter works with the law of diffusion going on pixel intensities. In medical images, edges in the image play key roles to diagnose precise disease that helps doctors for healthier treatment. In this filter, at every point, local image variation is calculated and neighbourhood pixel values are averaged. JiangtaoXu et al [19], proposed an edge-preserving diffusion method for suppressing Gaussian noise using anisotropic diffusion filter [AD] with semi adaptive threshold. This 


\section{AN EFFICIENT NOISE SEPARATION TECHNIQUE FOR REMOVAL OF GAUSSIAN AND MIXED NOISES IN MONOCHROME AND COLOR IMAGES}

technique overcomes the blurring edges and lines of an image and improves the ability to suppress noise when the noise level is high. SondesTebini et al [20], Anisotropic Diffusion (AD) is used to suppress the noise from an image with unvarying the important basic structural properties of an original image. Image blurring is a major disadvantage in AD. He applied an innovative mathematical function with an anisotropic approach for better noise reduction and keeping basic fine properties like edges and lines of an input image.

\section{iii.Bilateralfilter:}

Tomasi and manduchi [21], proposed a nonlinear filter called bilateral filter to suppress the additive noise from images. Unlike Gaussian filter, it smoothens the images by preserving the basic structure of the image. It de-noises the image with the computed weighted average of each pixel's neighborhood. Some of the areas where the bilateral filter can be used are texture suppression, dynamic range compression, and photograph enrichment. It is given by the product of two sub-kernels: gray-level kernel $M g$ and distance kernel $N d$. Gray level kernel: The distance of gray levels between an arbitrary pixel of intensity value $x(a 1, b 1)$ with respect to its central pixel of intensity value $x(a, b)$ is given by

$$
P_{g}=\left[\left|x^{2}\left(a_{1}, b_{1}\right)-x^{2}(a, b)\right|\right]^{1 / 2}
$$

The gray level sub kernel Mg is stated by:

$$
M_{g}=\exp \left(-\frac{1}{2}\left(\frac{P_{g}}{\sigma_{g}}\right)^{2}\right)
$$

Where $\sigma g$ is a distribution function for $M g$.

Distance kernel: The distance between any arbitrary pixel at location $(\mathrm{p} 1, \mathrm{q} 1)$ with respect to the center pixel at location $(\mathrm{p}, \mathrm{q})$ is termed as Euclidean distance and it is given by

$$
Q s=\sqrt{\left(p_{1}-p\right)^{2}+\left(q_{1}-q\right)^{2}}
$$

The geometric or distance sub-kernel is defined by

$$
N_{d}=\exp \left(-\frac{1}{2}\left(\frac{Q_{s}}{\sigma_{d}}\right)^{2}\right)
$$

At this juncture, $\sigma d$ is the standard deviation of $N d$. Now bilateral filter $M b$ is obtained by the Product of $M g$ and $N d$.

$$
\mathrm{Mb}=\mathrm{MgNd}
$$

Ju Zhang et al [22] proposed a new technique by amalgamating a wavelet transform and fast bilateral filter to de-speckle the US images to detect minor tumors and helping doctors to do precise diagnosis. KirtiV.Thakur et al [23], taken advantages of the bilateral filter into consideration designed a new technique called Poison

Reducing Bilateral Filter (PRBF) to remove the poison noise.

\section{iv.Non-local Means Filter:}

An elegant weighted average scheme called Non-Local Means (NLM) to de-noise the image has been proposed by Buades et al[24]. The simple idea is instead of looking at averages in the local neighborhood we are going to lookat averages in the whole image. Here a weighted averaging of pixel values centered at regions that are similar to the region centered at the estimated pixel values have been calculated. P.V. Sudeep[25], An unbiased nonlocal means method is implemented to reduce Speckle reduction in medical Ultra Sound images. It has two steps first, using ML Estimator estimate the shape and scale parameters and Compute the de-speckled value for every pixel in the image. Second, for each pixel in an image, compute the unbiased value using Unbiased NLM method. Gaihua Wang et al[26], An Improved Non-Local Means Filter(INLM) Algorithm has been proposed based on combining merits of NLM and BILF(bilateral) with added texture information as weights toquash the Gaussian noise in color images with retaining structural properties of a color image. It shows the performance when the variance of Gaussian and mixture noise is $0.01,0.02$ and 0.05 and shows poor performance when the variance of Gaussian and mixture noise is 0.03 and its computational time is more when compare with BILF filter.

\subsection{Transform Domain Filters(Wavelet):}

In this filtering, noisy image is transformed into discrete form and then there apply de-noising filters in the renovated image. These are classified as Curvelet filter, Data-adaptive and Non-data Adaptive filters (Wavelet Domain), Nonlocal based (Block Matching 3D-BM3D, Block Matching 4D-BM 4D) and Contourlet filter. Wavelet filters are frequently applied to reduce the noise from an image with preservative basic fine points of the original image. The widely used Wavelet domain filters are Stein's unbiased risk estimate (SURE), Bayes, Bivariate, Non-local mean, shrinkage rulesbased filters.

\section{I.VISU shrink:}

Visushrink is thresholding by applying the Universal threshold suggested by Donoho and Johnstone [27]. This threshold value is given by $\sigma \sqrt{2 \log \mathrm{A}}$ here $\sigma$ represents the variance in noise and A represents a number of pixels in the image. It deals with additive noise. This method cannot remove the speckle noise.

\section{II.Stein's unbiased risk estimate (SURE) shrink:}

This is designed by incorporating the universal and sure threshold. Its purpose is to diminish the Mean Squared Error. Thierry Bluet al [28], introduced a novel method to de-noise the image, based on the image-domain and minimizing an estimate of mean squared error. Zhang et al [29], has proposed a new hybrid thresholding function to restore the original image by merging local wiener filter

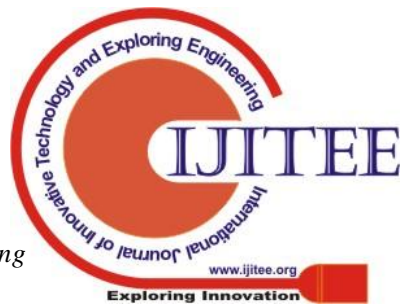


with pointwise thresholding function. Xiaobo Zhang et al[30], improved the SURE-LET method by using wiener filters and suggested an enhanced inter-scale based SURELET for better de-noising performance by combining the wiener estimator and the inter-scale based SURE-LET thresholding function.

\section{III.BAYES shrink:}

Bayes shrink [31] threshold is used to minimize the Bayesian risk. Synthetic Aperture Radar (SAR) images are degraded with multiplicative speckle noise. AlinAchimee al [32], proposed a novel approach based on Bayesian algorithm with wavelet analysis framework to de-speckle SAR images with unvarying basic fine details and texture information. IsratZahanNishu et al [33] developed a novel method to de-speckle noise from images using Speckle Reducing Anisotropic Diffusion (SRAD) with a grouping of Discrete Wavelet Transform (DWT) using Bayesian thresholding. An exponential transformation is applied to improve the image contrast and later SRAD filter is applied on log compressed image which preserves the basic fine details of the image while suppressing the noise without removing the edge and line details. The log transformation is applied to a de-noised image. Finally, apply DWT using Bayesian thresholding to get the high visual quality denoised image.

\subsection{De-noising Convolution Neural Networks (DnCNN)(Neural Networks):}

To optimize and automate the procedure of suppressing noise from the image novel filters called Neural Network (NN) filters are applied. To quash the noise in images a built-in pre-trained neural network called DnCNN is used. Yuxing Zhao et al [34], has proposed a new technique called improved feed-forward DnCNN to remove random noise in desert seismic data. Kai Zhang et al [35] implicitly removes the latent clean image from hidden layers using residual learning approach. It motivates to train a single DnCNN model to tackle Gaussian de-noising, Single image superresolution, and Image de-blocking. Hamid Reza Shahdoosti [36] has proposed a new approach Deep Conventional Neural Networks DCNN) to de-noise grayscale and Ultrasound images degraded with different noises by preserving basic structures of an original image. First, noisy image is broken down into low and high-frequency subbands by using non-sub sampled sherlet transforms. Then, 2D block's high-frequency subbands are combined to form 3D blocks. After that, the trained network is able to determine whether the noisy patch in NSST (nonsubsampled sherlet transform) domain belongs to the edgerelated class or not. And then the Coefficients which do not belong to edge related class are de-noised with Shrinkage method using Adaptive threshold. This algorithm is not suitable for color images. It takes more running time when compared with Adaptive Total Variance model, Stein's unbiased risk estimate (SURE), Bivariate, Block matching 3D and another state- of-art-algorithms. The below figure shows, the process of de-noising using a trained network called Convolutional Neural Network (CNN).

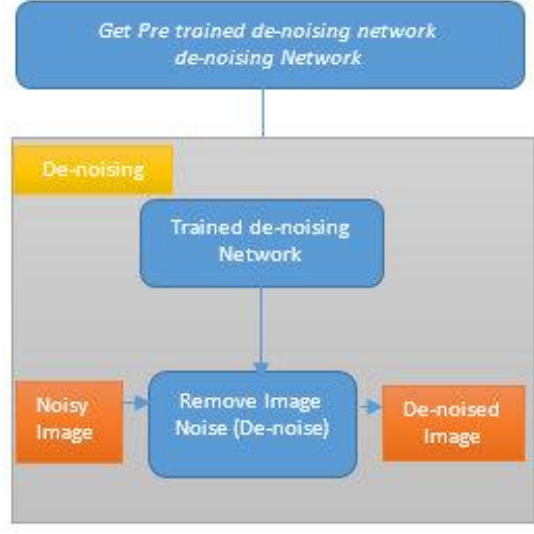

Fig 7: De-noising Process using Convolutional Neural Network

\section{4 QUANTITATIVE PERFORMANCE EVALUATION OF DE-NOISING FILTERS:}

\subsection{Peak Signal to Noise Ratio (PSNR):}

PSNR is considered as performance metrics in de-noising images and it is measured in $\mathrm{dB}$. It can be deliberated by estimating the pixel difference between the initial image and the de-noised image. It is given by

$P S N R=20 \log _{10}\left(\frac{M A X(\mathrm{a})}{\sqrt{M S E}}\right) \mathrm{dB}$

Here MAX (a) represents maximum signal value occurs in the input image. The increased PSNR quantifies the quality of the output image.

\subsection{Mean Squared Error (MSE):}

It is used to assess the quality of the de-noised image with respect to its original image. MSE is calculated by averaging the squared intensity of the real input image pixel values and the resultant output image pixels values. It can be calculated as follows.

$$
\begin{aligned}
& M S E=\frac{1}{r \times c}\left\|f-f^{1}\right\|^{2} \\
& =\frac{1}{r \times c} \sum_{p=1}^{r} \sum_{q=1}^{c}\left(f_{p q}-f_{p q}^{1}\right)^{2}
\end{aligned}
$$

Here $\mathrm{f}$ represents the matrix data of input image, $f$ signifies the matrix data of the noisy image, $r$ signifies rows of pixels values in an image and $\mathrm{p}$ specifies row index, $\mathrm{c}$ represents columns of pixel values in an image and $\mathrm{q}$ is column index. It is required that MSE values should be less for a perfect de-noised image.

\subsection{Structural Similarity (SSIM) Index:}

SSIM is the eminent quality metric applied to compute the perceptual difference between two identical images. The SSIM is computed with modelling any image distortion as a mishmash of three factors that are forfeiture of correlation, 


\section{AN EFFICIENT NOISE SEPARATION TECHNIQUE FOR REMOVAL OF GAUSSIAN AND MIXED NOISES IN MONOCHROME AND COLOR IMAGES}

luminance distortion, and contrast distortion. This can be given by

$$
S S I M=\frac{\left(2 \mu_{x 1} \mu_{x 2}+k_{1}\right)\left(2 \sigma_{x 1 x 2}+k_{2}\right)}{\left(\mu_{x 1}{ }^{2}+\mu_{x 2}{ }^{2}+k_{1}\right)\left(\sigma_{x 1}{ }^{2}+\sigma_{x 2}{ }^{2}+k_{2}\right)}
$$

Where, $\mu x 1$ represents the average of $x 1, \mu x 2$ specifies the average of $x 2, \sigma x 1$ is the variance of $x 1$, and $\sigma \times 2$ is the variance of $x 2$ and $\sigma x 1 \times 2$ is the co-variance of $x 1 ; x 2: k 1 ; k 2$ are variables.

\subsection{Entropy:}

It is applied to characterize the texture of an input image. It is defined as

$$
-\sum m^{*} \log 2(m)
$$

Where m represents the histogram counts.

\section{RESULTS AND DISCUSSION:}

In the present investigation, two images are taken one in grayscale and another in color image. For grayscale, cameraman.tif image is taken with dimensions $512 \times 512$. And for a color image, lena.png image is taken with dimensions 512x512. An additive Gaussian noise of different variance viz., $0.01,0.03$ and 0.05 is added to cameraman.tif. The metrics such as PSNR, MSE, SSIM, FSIM, EPI, and Entropy are computed for both the noisy and de-noised images. The de-noised image is attained by applying various filters such as Non-Local Means, Bilateral, Gaussian filter, anisotropic, Guided filter,wiener2, VISU shrink soft Thresholding, VISU Shrink hard thresholding and DnCNN. The above-said performance metric values of different filters applied to the grayscale image with single noise are tabulated in table1. From the table1 and graph (Fig 8 ) it is evident that the DnCNN filter is best performing by having higher PSNR of 28.60, 25.70, 24.03 and the lower MSE value of $89.85,175.12$, and 257.12 for the variance $0.01,0.03$ and 0.05 respectively.

Likewise in terms of SSIM, FSIM, EPI, and Entropy also DnCNN is performing best and the values are highlighted in the table1. To have better evidence of the same instead of single noise (Gaussian), all the metrics have been computed by adding mixed noises of Gaussian, impulsive and speckle noise for the grayscale cameraman.tif. The same is tabulated in table 2 for comparison. It is evident from the table 2 and graph (Fig 8) the DnCNN filter performs well in de-noising an image with mixed noise also. The highest PSNR and lowest MSE which is highlighted in the table shows its best performance among all other filters.

The Fig 10, Fig11, Fig12 depicts the outputs of various filters which were discussed in section 3 for a single noise grayscale image. The Fig13, Fig14, Fig15 depicts the outputs of several filters for a grayscale image with mixed noise. The same procedure was followed and extrapolated for color images. Both single noise and mixed noise is taken into account for de-noising. The filters such as NLM, BILF, Gaussian, AF, Guided, VISU soft and hard shrink threshold and $\mathrm{DnCNN}$ are considered to de-noise the image. The performance of these filters was assessed using the metrics viz., PSNR, MSE, SSIM, FSIM. Table 3 and Table 4 shows the values obtained for single noise and mixed noise. It is evident from the tables and graphs (Fig 9) the DnCNN filter is performing best with high PSNR, SSIM, and lowest MSE. As far as to feature similarity concerns in color image, the bilateral filter works well. It is shown in table 4.

The Fig16, Fig17, Fig18 depicts the outputs of applying various filters for a color image with a single noise. The

Fig19, Fig20, Fig21 depicts the outputs of applying various filters for a color image with mixed noises.

Table 1 Performance (Single noise) of various methods as measured by PSNR, MSE, SSIM, Entropy, FSIM, and EPI

\begin{tabular}{|c|c|c|c|c|c|c|c|c|c|c|c|c|c|c|c|c|c|c|}
\hline \multicolumn{19}{|c|}{ Gray Scale Image-Guassian Noise } \\
\hline \multirow[b]{2}{*}{ Filters/Metrics } & \multicolumn{6}{|c|}{ Mean 0, Variance 0.01} & \multicolumn{6}{|c|}{ Mean 0 , Variance 0.03} & \multicolumn{6}{|c|}{ Mean 0 , Variance 0.05} \\
\hline & PSNR & MSE & SSIM & Entropy & FSIM & EPI & PSNR & MSE & SSIM & Entropy & FSIM & EPI & PSNR & MSE & SSIM & Entropy & FSIM & EPI \\
\hline Noisy Image & 20.37 & 567.27 & 0.34 & 7.53 & 0.69 & 0.47 & 15.88 & 1678.02 & 0.21 & 7.60 & 0.57 & 0.46 & 13.9 & 2646.85 & 0.17 & 7.52 & 0.51 & 0.46 \\
\hline Non Local Means & 28.07 & 101.40 & 0.79 & 6.90 & 0.87 & 0.71 & 24.61 & 225.08 & 0.67 & 6.94 & 0.83 & 0.70 & 22.67 & 357.38 & 0.58 & 6.95 & 0.79 & 0.52 \\
\hline Bilateral Filter & 26.17 & 157.22 & 0.61 & 7.23 & 0.85 & 0.56 & 22.02 & 407.94 & 0.41 & 7.43 & 0.75 & 0.56 & 20.01 & 648.33 & 0.32 & 7.49 & 0.70 & 0.44 \\
\hline Gaussian Filter & 24.79 & 215.84 & 0.66 & 7.24 & 0.81 & 0.63 & 22.86 & 336.61 & 0.49 & 7.38 & 0.74 & 0.63 & 21.56 & 453.74 & 0.42 & 7.43 & 0.70 & 0.51 \\
\hline $\begin{array}{l}\text { Anisotropic } \\
\text { Diffusion Filter }\end{array}$ & 26.90 & 132.90 & 0.74 & 7.09 & 0.87 & 0.61 & 23.09 & 319.32 & 0.55 & 7.25 & 0.81 & 0.62 & 21.05 & 510.86 & 0.44 & 7.32 & 0.76 & 0.43 \\
\hline Guided Filter & 24.48 & 231.82 & 0.51 & 7.36 & 0.81 & 0.51 & 18.14 & 998.52 & 0.26 & 7.68 & 0.63 & 0.50 & 15.50 & 1831.35 & 0.19 & 7.47 & 0.56 & 0.44 \\
\hline Wiener2 Filter & 26.18 & 156.86 & 0.62 & 7.30 & 0.83 & 0.53 & 22.03 & 407.40 & 0.42 & 7.49 & 0.72 & 0.53 & 20.11 & 633.66 & 0.33 & 7.58 & 0.66 & 0.43 \\
\hline $\begin{array}{l}\text { VISU Shrink(Soft } \\
\text { thresholding) }\end{array}$ & 24.38 & 237.30 & 0.71 & 7.20 & 0.74 & 0.52 & 21.98 & 412.57 & 0.61 & 7.20 & 0.75 & 0.52 & 20.38 & 595.86 & 0.34 & 7.26 & 0.73 & 0.44 \\
\hline $\begin{array}{l}\text { VISU Shrink(Hard } \\
\text { thresholding) }\end{array}$ & 24.22 & 246.01 & 0.57 & 7.25 & 0.78 & 0.49 & 20.59 & 567.93 & 0.40 & 7.51 & 0.70 & 0.49 & 15.24 & 1946.19 & 0.19 & 7.74 & 0.62 & 0.43 \\
\hline DnCNN & 28.60 & 89.85 & 0.81 & 7.03 & 0.89 & 0.73 & 25.70 & 175.12 & 0.71 & 7.05 & 0.85 & 0.53 & 24.03 & 257.12 & 0.63 & 7.11 & 0.82 & 0.57 \\
\hline
\end{tabular}
corresponding to varying noise levels

Table 2 Performance (mixed noise) of various methods as measured by PSNR, MSE, SSIM, Entropy, FSIM, EPI corresponding to varying noise levels 


\begin{tabular}{|c|c|c|c|c|c|c|c|c|c|c|c|c|c|c|c|c|c|c|}
\hline \multicolumn{19}{|c|}{ Gray Scale Image-Mixed Noise } \\
\hline \multirow[b]{2}{*}{ Filters/Metrics } & \multicolumn{6}{|c|}{ Mean 0, Variance 0.01} & \multicolumn{6}{|c|}{ Mean 0 , Variance 0.03} & \multicolumn{6}{|c|}{ Mean 0, Variance 0.05} \\
\hline & PSNR & MSE & SSIM & Entropy & FSIM & EPI & PSNR & MSE & SSIM & Entropy & FSIM & EPI & PSNR & MSE & SSIM & Entropy & FSIM & EPI \\
\hline Noisy Image & 18.37 & 947.27 & 0.29 & 7.59 & 0.65 & 0.45 & 13.98 & 2598.95 & 0.17 & 7.50 & 0.79 & 0.40 & 12.53 & 3895.24 & 0.13 & 7.29 & 0.46 & 0.39 \\
\hline Non Local Means & 26.53 & 144.63 & 0.74 & 6.90 & 0.85 & 0.64 & 22.84 & 337.94 & 0.59 & 6.93 & 0.71 & 0.51 & 20.88 & 531.56 & 0.50 & 6.99 & 0.75 & 0.44 \\
\hline Bilateral Filter & 23.18 & 312.86 & 0.52 & 7.26 & 0.81 & 0.52 & 20.06 & 641.25 & 0.33 & 7.46 & 0.70 & 0.43 & 18.47 & 924.32 & 0.26 & 7.52 & 0.64 & 0.4 \\
\hline Gaussian Filter & 24.11 & 252.39 & 0.59 & 7.29 & 0.78 & 0.58 & 21.73 & 437.08 & 0.43 & 7.40 & 0.75 & 0.46 & 20.14 & 628.99 & 0.35 & 7.44 & 0.65 & 0.41 \\
\hline $\begin{array}{l}\text { Anisotropic } \\
\text { Diffusion Filter }\end{array}$ & 23.10 & 318.76 & 0.61 & 7.09 & 0.82 & 0.53 & 19.92 & 661.78 & 0.44 & 7.26 & 0.57 & 0.34 & 18.34 & 952.34 & 0.32 & 7.19 & 0.71 & 0.28 \\
\hline Guided Filter & 21.45 & 466.16 & 0.40 & 7.49 & 0.74 & 0.48 & 15.64 & 1776.29 & 0.20 & 7.75 & 0.67 & 0.40 & 13.40 & 2974.91 & 0.14 & 7.74 & 0.5 & 0.39 \\
\hline Wiener2 Filter & 23.40 & 297.44 & 0.51 & 7.37 & 0.78 & 0.5 & 19.80 & 676.98 & 0.33 & 7.55 & 0.66 & 0.41 & 18.30 & 960.85 & 0.26 & 7.61 & 0.61 & 0.38 \\
\hline $\begin{array}{l}\text { VISU Shrink(Soft } \\
\text { thresholding) }\end{array}$ & 22.39 & 375.00 & 0.65 & 7.19 & 0.74 & 0.48 & 21.06 & 508.96 & 0.48 & 7.25 & 0.73 & 0.39 & 19.58 & 716.79 & 0.33 & 7.32 & 0.68 & 0.35 \\
\hline $\begin{array}{l}\text { VISU Shrink(Hard } \\
\text { thresholding) }\end{array}$ & 23.15 & 315.18 & 0.59 & 7.30 & 0.76 & 0.43 & 17.43 & 1175.69 & 0.25 & 7.71 & 0.61 & 0.44 & 14.60 & 2253.98 & 0.16 & 7.85 & 0.52 & 0.36 \\
\hline DnCNN & 27.13 & 126.01 & 0.76 & 7.06 & 0.87 & 0.68 & 24.00 & 258.98 & 0.62 & 7.07 & 0.81 & 0.55 & 21.78 & 431.84 & 0.51 & 7.12 & 0.76 & 0.48 \\
\hline
\end{tabular}

Table 3 Performance (single noise) of various methods as measured by PSNR, MSE, SSIM, FSIM corresponding to varying noise levels

\begin{tabular}{|l|c|c|c|c|c|c|c|c|c|c|c|c|}
\hline \multicolumn{10}{|c|}{ Color Image -Guassian Noise } \\
\hline \multicolumn{1}{|c}{ Filters/Metrics } & \multicolumn{10}{|c|}{ Mean 0, Noise Variance 0.01 } & \multicolumn{1}{c|}{ Mean 0, Noise Variance 0.03 } & \multicolumn{3}{c|}{ Mean 0, Noise Variance 0.05 } \\
\cline { 2 - 13 } & PSNR & MSE & SSIM & FSIM & PSNR & MSE & SSIM & FSIM & PSNR & MSE & SSIM & FSIM \\
\hline Noisy Image & 20.22 & 618.29 & 0.80 & 0.74 & 15.78 & 1716.81 & 0.60 & 0.80 & 13.85 & 2680.09 & 0.49 & 0.75 \\
\hline Non Local Means & 23.65 & 28.57 & 0.90 & 0.88 & 20.13 & 631.05 & 0.81 & 0.90 & 18.63 & 892.12 & 0.75 & 0.88 \\
\hline Bilateral Filter & 29.97 & 65.51 & 0.97 & 0.88 & 25.25 & 194.32 & 0.93 & 0.91 & 22.49 & 366.47 & 0.87 & 0.88 \\
\hline Gaussian Filter & 28.85 & 84.76 & 0.97 & 0.85 & 25.35 & 189.85 & 0.93 & 0.88 & 23.49 & 291.18 & 0.89 & 0.85 \\
\hline $\begin{array}{l}\text { Anisotropic } \\
\text { Diffusion Filter }\end{array}$ & 23.04 & 322.90 & 0.89 & 0.75 & 16.49 & 1458.82 & 0.64 & 0.81 & 14.21 & 2463.72 & 0.51 & 0.75 \\
\hline Guided Filter & 24.99 & 206.07 & 0.92 & 0.79 & 18.24 & 975.30 & 0.72 & 0.85 & 15.57 & 1803.12 & 0.58 & 0.79 \\
\hline $\begin{array}{l}\text { IISU Shrink(Soft } \\
\text { thresholding) }\end{array}$ & 20.21 & 619.34 & 0.80 & 0.86 & 24.81 & 215.06 & 0.94 & 0.86 & 23.89 & 265.60 & 0.93 & 0.86 \\
\hline $\begin{array}{l}\text { VISU Shrink(Hard } \\
\text { thresholding) }\end{array}$ & 20.87 & 532.32 & 0.82 & 0.84 & 22.51 & 364.61 & 0.87 & 0.89 & 18.41 & 936.68 & 0.73 & 0.84 \\
\hline DnCNN & 30.83 & 24.22 & 0.98 & 0.91 & 27.81 & 107.58 & 0.96 & 0.93 & 26.04 & 161.84 & 0.94 & 0.91 \\
\hline
\end{tabular}

Table 4 Performance (mixed noise) of various methods as measured by PSNR, MSE, SSIM, FSIM corresponding to varying noise levels

\begin{tabular}{|c|c|c|c|c|c|c|c|c|c|c|c|c|}
\hline \multicolumn{13}{|c|}{ Color Image -Mixed Noise } \\
\hline \multirow{2}{*}{ Filters/Metrics } & \multicolumn{4}{|c|}{ Mean 0, Variance 0.01} & \multicolumn{4}{|c|}{ Mean 0, Variance 0.03} & \multicolumn{4}{|c|}{ Mean 0, Variance 0.05} \\
\hline & PSNR & MSE & SSIM & FSIM & PSNR & MSE & SSIM & FSIM & PSNR & \begin{tabular}{|l|} 
MSE \\
\end{tabular} & SSIM & FSIM \\
\hline Noisy Image & 18.30 & 961.20 & 0.73 & 0.86 & 13.97 & 2607.23 & 0.49 & 0.75 & 12.13 & 3978.51 & 0.38 & 0.69 \\
\hline Non Local Means & 21.48 & 462.74 & 0.85 & 0.92 & 18.33 & 955.11 & 0.73 & 0.86 & 17.07 & 1275.83 & 0.66 & 0.83 \\
\hline Bilateral Filter & 26.91 & 132.39 & 0.95 & 0.94 & 22.04 & 406.57 & 0.86 & 0.88 & 19.38 & \begin{tabular}{|l|}
749.26 \\
\end{tabular} & 0.76 & 0.83 \\
\hline Gaussian Filter & 27.33 & 120.27 & 0.95 & 0.92 & 23.17 & 313.39 & 0.89 & 0.84 & 21.03 & 513.32 & 0.83 & 0.79 \\
\hline Anisotropic Diffusion Filter & 19.90 & 665.32 & 0.79 & 0.88 & 14.38 & 2372.72 & 0.52 & 0.75 & 12.35 & 3788.71 & 0.39 & 0.69 \\
\hline & 21.80 & 429.24 & 0.85 & 0.91 & 15.72 & 1742.41 & 0.59 & 0.79 & 13.36 & 3001.39 & 0.44 & 0.72 \\
\hline $\begin{array}{l}\text { VISU Shrink(Soft } \\
\text { thresholding) }\end{array}$ & 24.93 & 208.89 & 0.95 & 0.86 & 14.30 & 2417.76 & 0.51 & 0.85 & 21.10 & 504.39 & 0.87 & 0.84 \\
\hline $\begin{array}{l}\text { VISU Shrink(Hard } \\
\text { thresholding) }\end{array}$ & 25.30 & 191.81 & 0.93 & 0.91 & 13.97 & 2604.36 & 0.49 & 0.84 & 15.11 & 2005.07 & 0.54 & 0.76 \\
\hline $\mathrm{DnCNN}$ & 28.87 & 84.37 & 0.97 & 0.95 & 25.22 & 196.51 & 0.94 & 0.91 & 22.69 & 349.73 & 0.89 & 0.88 \\
\hline
\end{tabular}




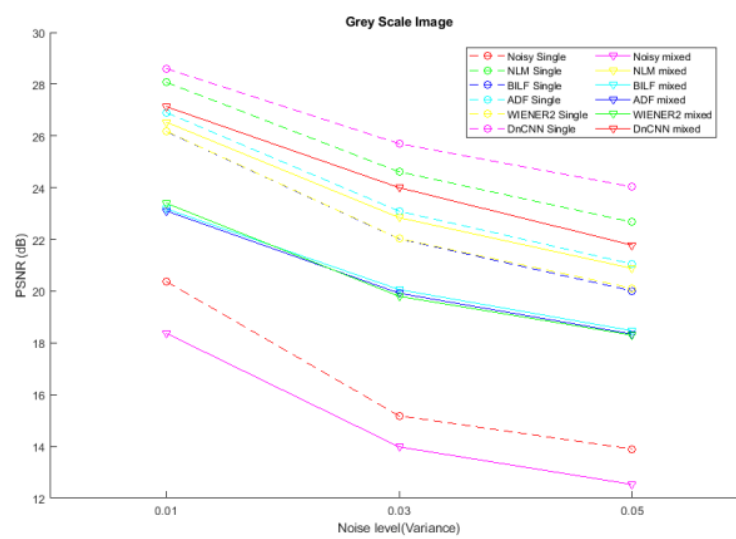

a)PSNR

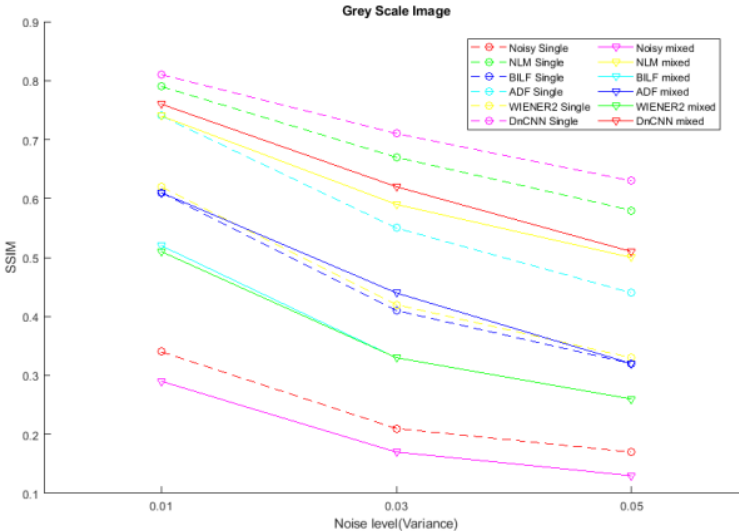

c) SSIM

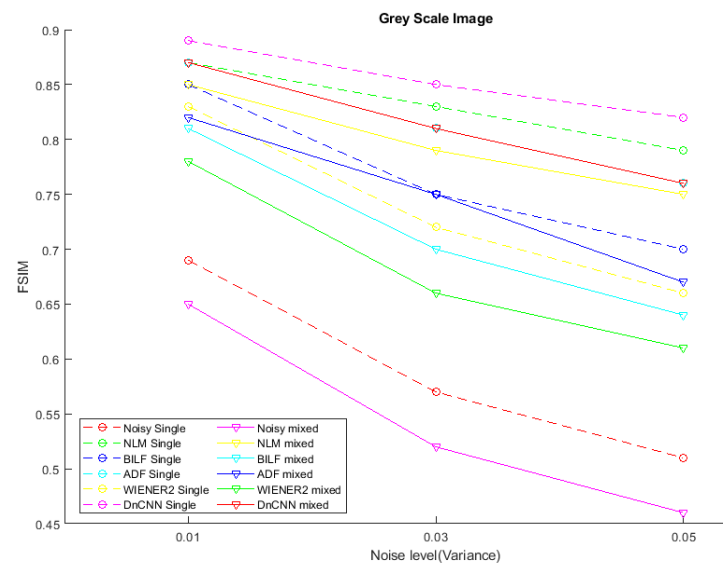

e) FSIM

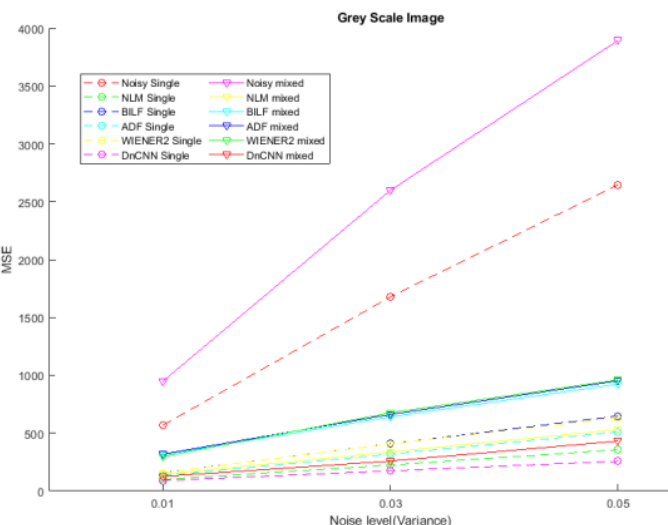

b) MSE

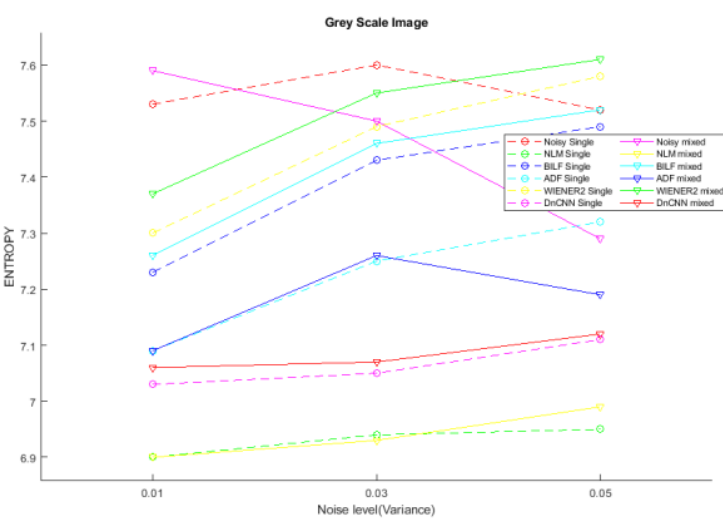

d) ENTROPY

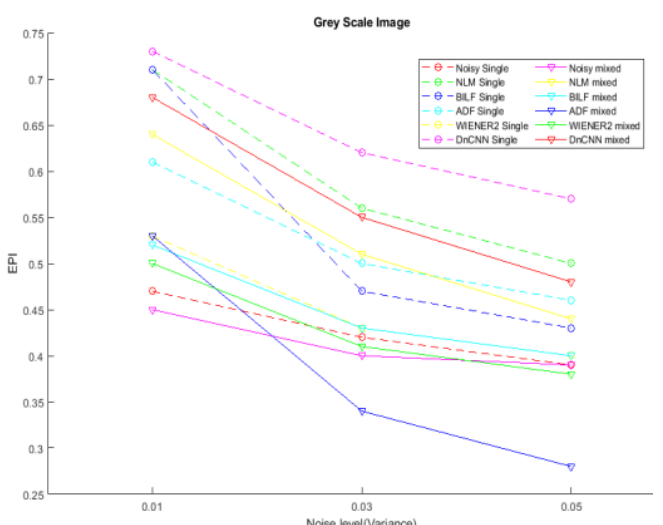

f)EPI

Fig. 8 Performance (grayscale) graph of various methods as measured by PSNR, MSE, SSIM, Entropy, FSIM and EPI corresponding to varying noise levels (Single and mixed noises) 


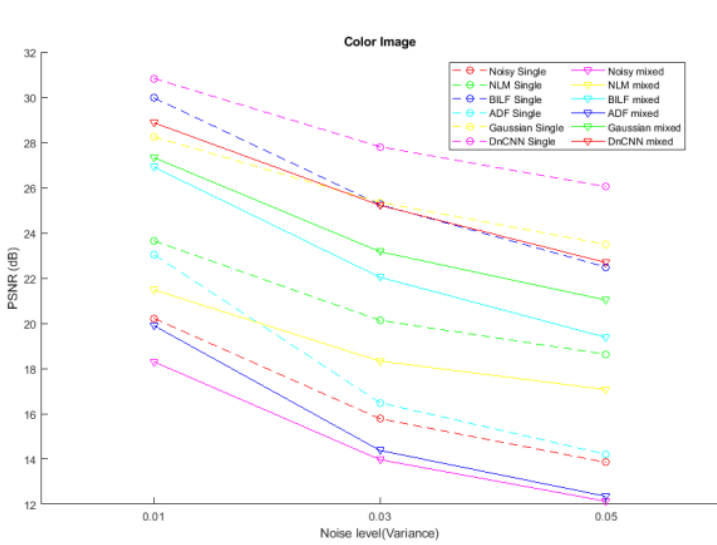

a)PSNR

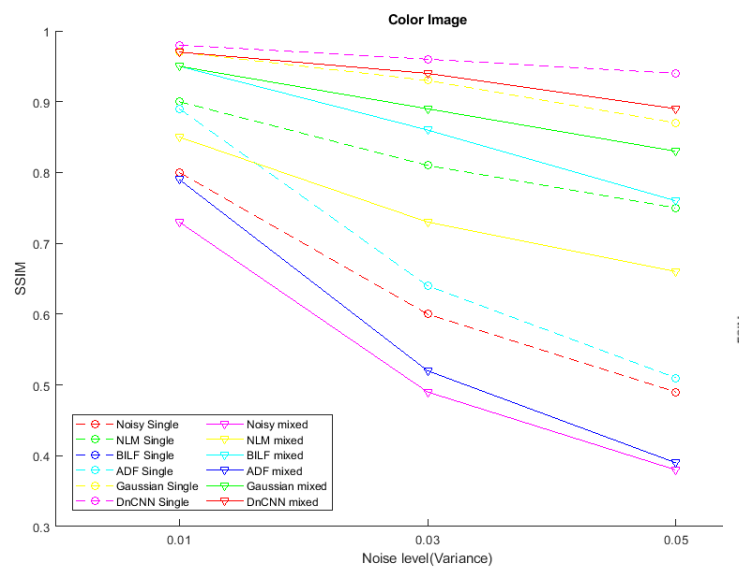

c) SSIM

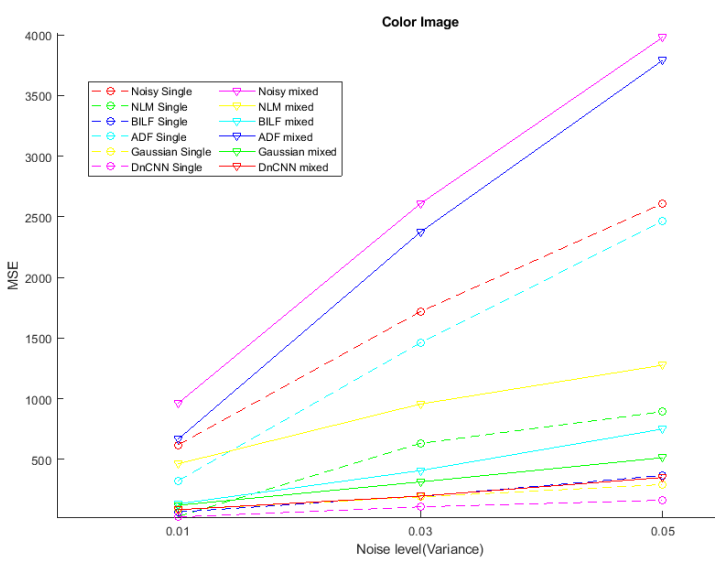

b)MSE

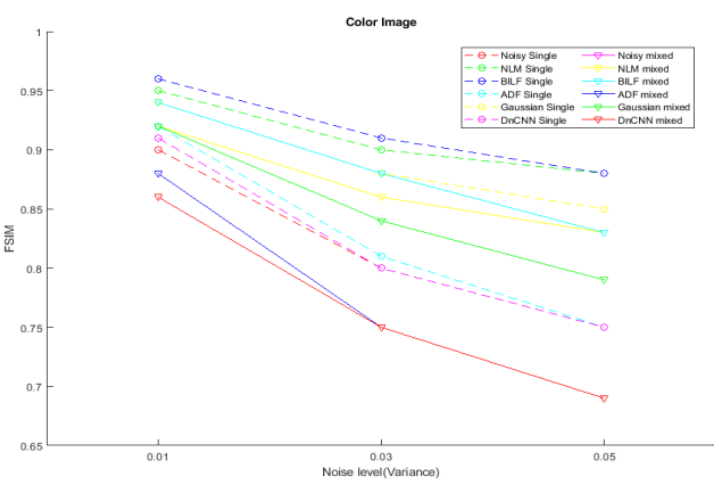

d) FSIM

Fig. 9 Performance (color) graph of various methods as measured by PSNR, MSE, SSIM and FSIM corresponding to varying noise levels (Single and mixed noises).

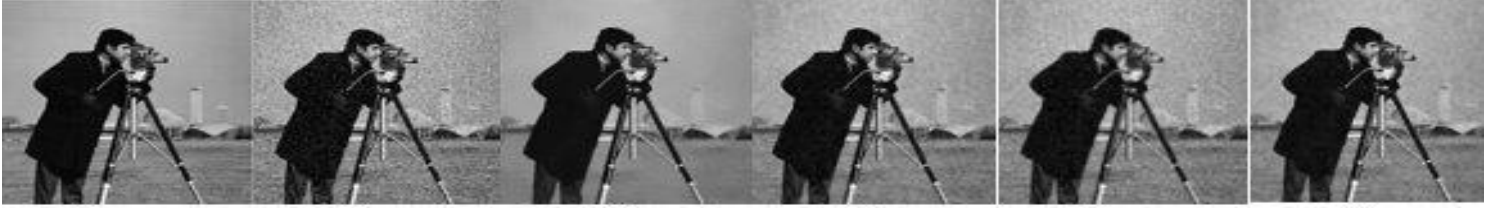

(a) (b) (c)

(e)

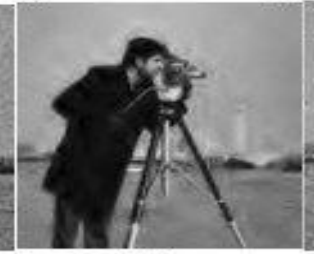

(i)

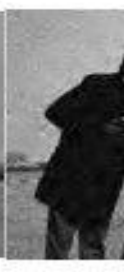

(h)

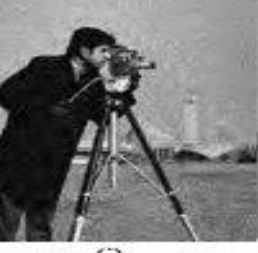

(j) (f)

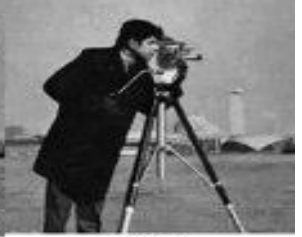

(k)

Fig. 10 a) Original Gray Image b)Single Noisy Image (mean 0, variance 0.01) c) NLM d) BILF e) Gaussian f)ADF g) Guided h)Wiener2 i) Visu-Shrink SURE(Soft threshold) j) Visu-Shrink SURE(Hard threshold) k)DnCNN

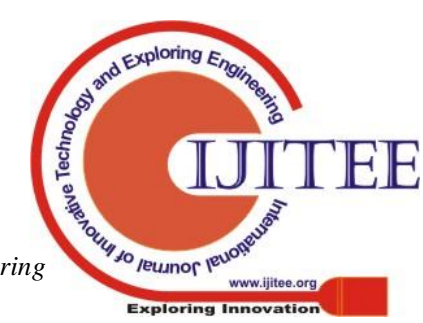




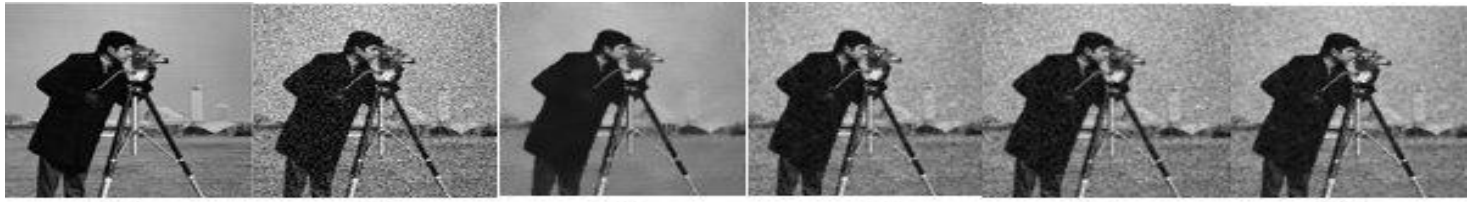

(a)

(b)

(c)

(d)

(e)

(f)

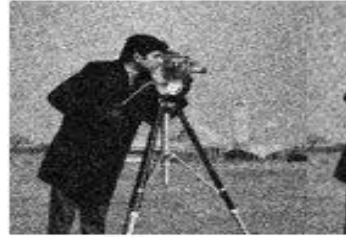

(g)

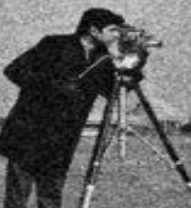

(h)

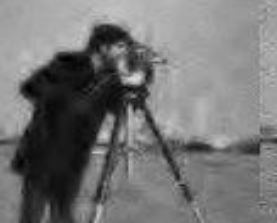

(i)

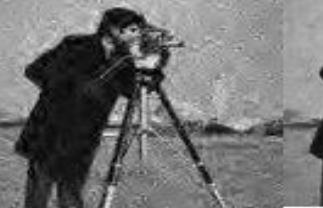

(j)

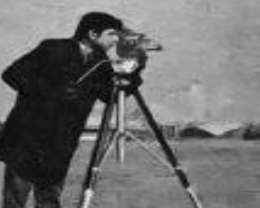

(k)

Fig. 11 a) Original Gray Image b)Single Noisy Image (mean 0, variance 0.03) c) NLM d) BILF e) Gaussian f)ADF g) Guided h)Wiener2 i) Visu-Shrink SURE(Soft threshold) j) Visu-Shrink SURE(Hard threshold) k)DnCNN

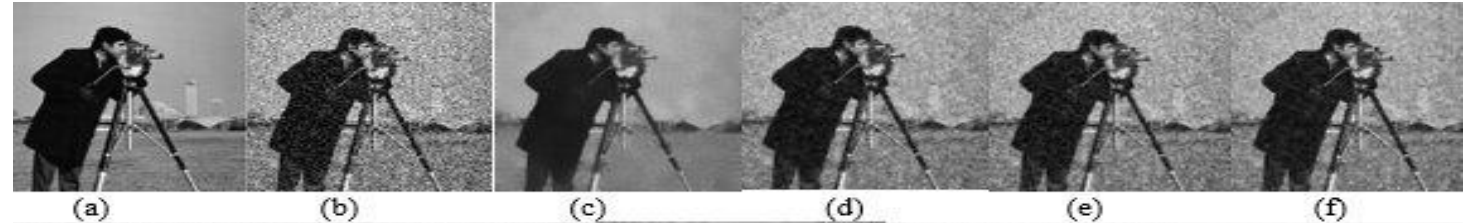

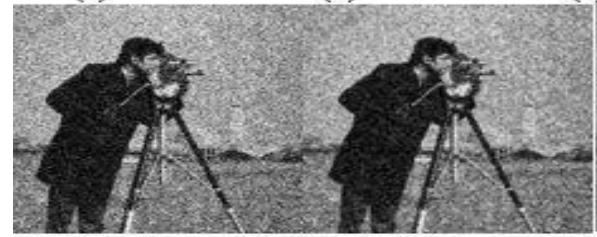

(g)

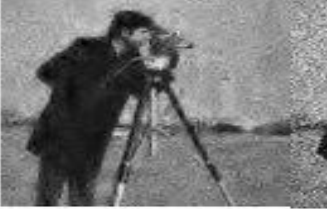

(i)

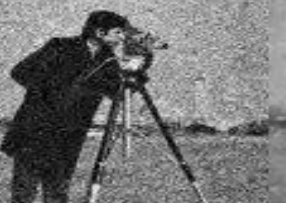

(j)

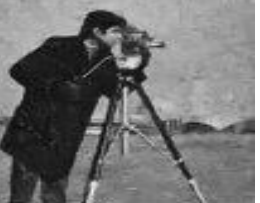

(k)

Fig. 12 a) Original Gray Image b)Single Noisy Image (mean 0, variance 0.05) c) NLM d) BILF e) Gaussian f)ADF g) Guided h)Wiener2 i) Visu-Shrink SURE(Soft threshold) j) Visu-Shrink SURE(Hard threshold) k)DnCNN

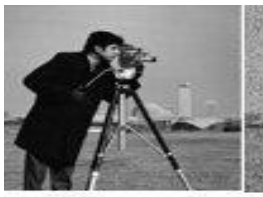

(a)

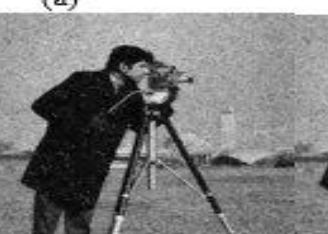

(g)

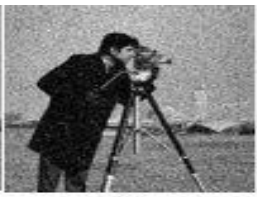

(b)

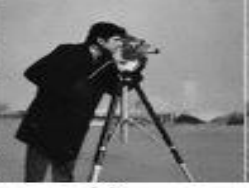

(c)

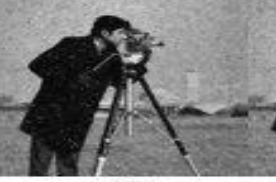

(d)

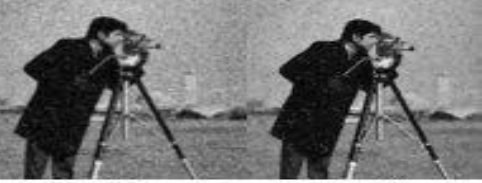

(e)

(f)

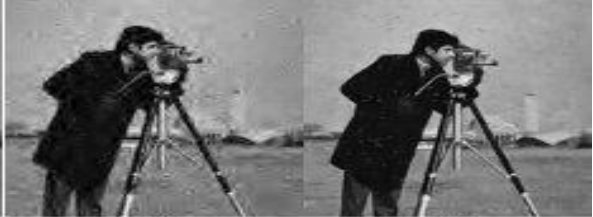

(k)

Fig. 13 a) Original Gray Image b)Mixed Noisy Image (mean 0, variance 0.01) c) NLM d) BILF e) Gaussian f)ADF g) Guided h)Wiener2 i) Visu-Shrink SURE(Soft threshold) j)Visu-Shrink SURE(Hard threshold) k)DnCNN

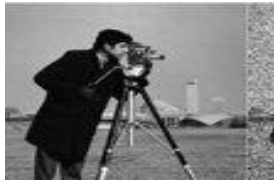

(a)

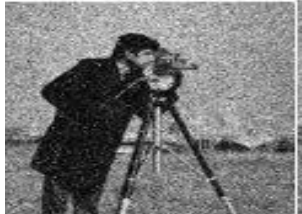

(g)

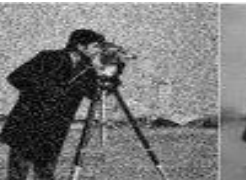

(b)

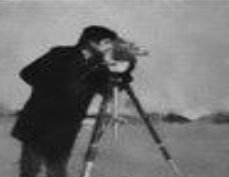

(c)

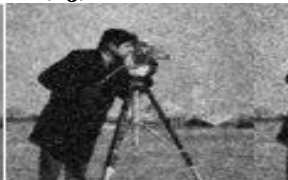

(d)

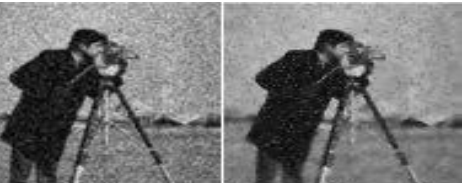

(e)

(f)

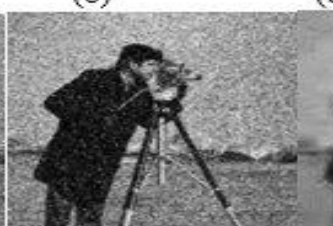

(h)

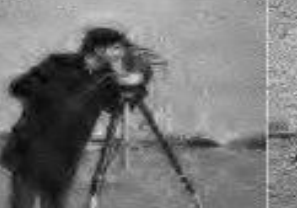

(i)

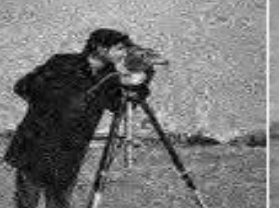

(j)

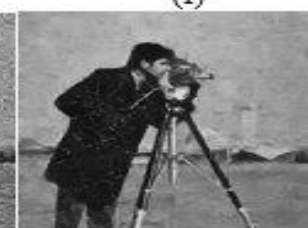

(k)

Fig. 14 a) Original Gray Image b)Mixed Noisy Image (mean 0, variance 0.03) c) NLM d) BILF e) Gaussian f)ADF g)

Guided h)Wiener2 i) Visu-Shrink SURE(Soft threshold) j) Visu-Shrink SURE(Hard threshold) k)DnCNN

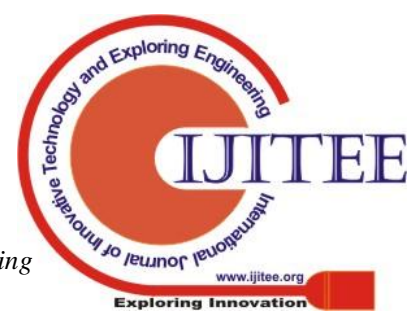




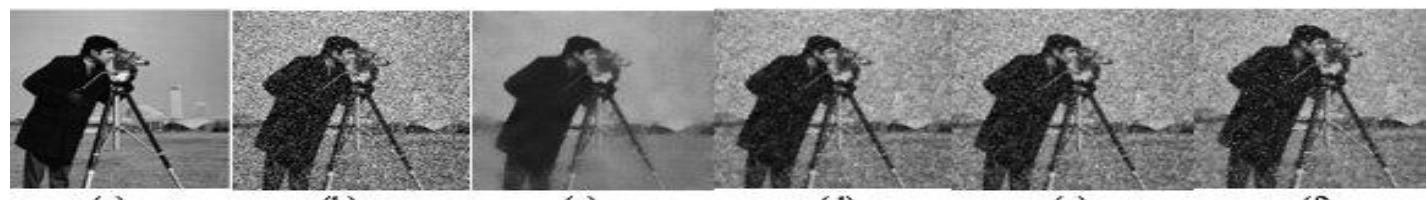

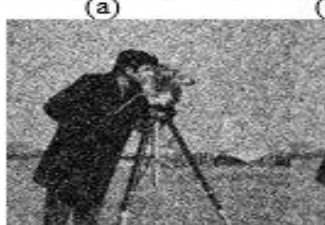

(g) (b)

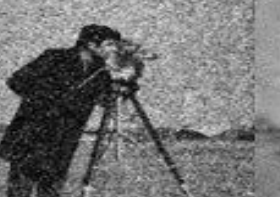

(h)

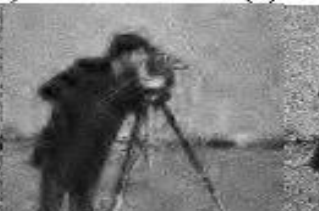

(i) (e)

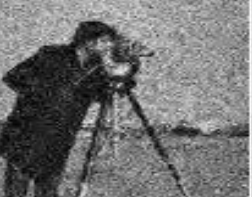

(j) (f)

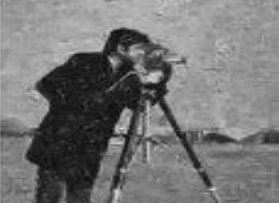

(k)

Fig. 15 a) Original Gray Image b)Mixed Noisy Image (mean 0, variance 0.05) c) NLM d) BILF e) Gaussian f)ADF g)

Guided h)Wiener2 i) Visu-Shrink SURE(Soft threshold) j) Visu-Shrink SURE(Hard threshold) k)DnCNN

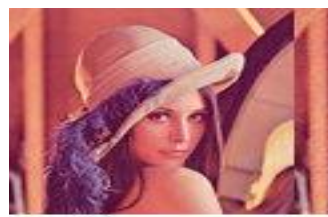

(a)

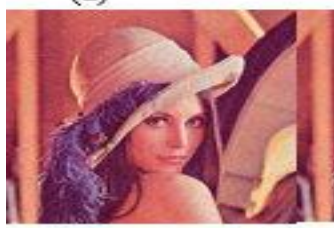

(f)

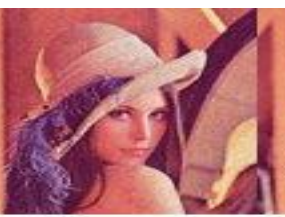

(b)

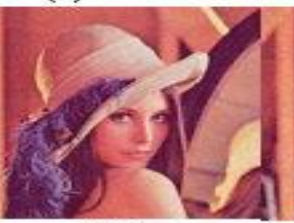

(g)

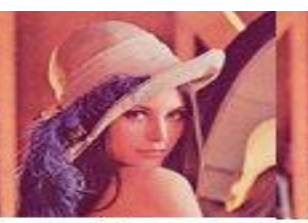

(c)

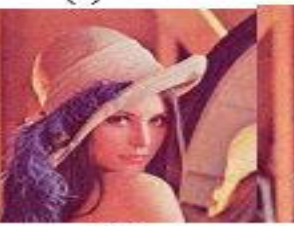

(h)

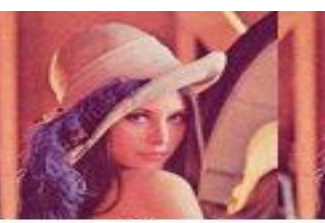

(d)

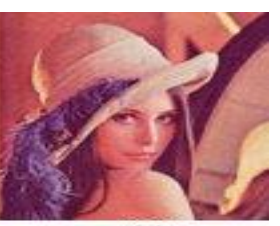

(e)

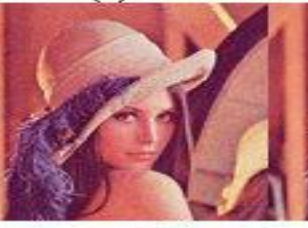

(i)

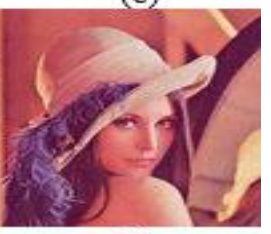

(j)

Fig. 16 a) Original Color Image b)Single Noisy Image (mean 0, variance 0.01) c) NLM d) BILF e) Gaussian f)ADF g) Guided h) Visu-Shrink SURE(Soft threshold) i) Visu-Shrink SURE(Hard threshold) j)DnCNN

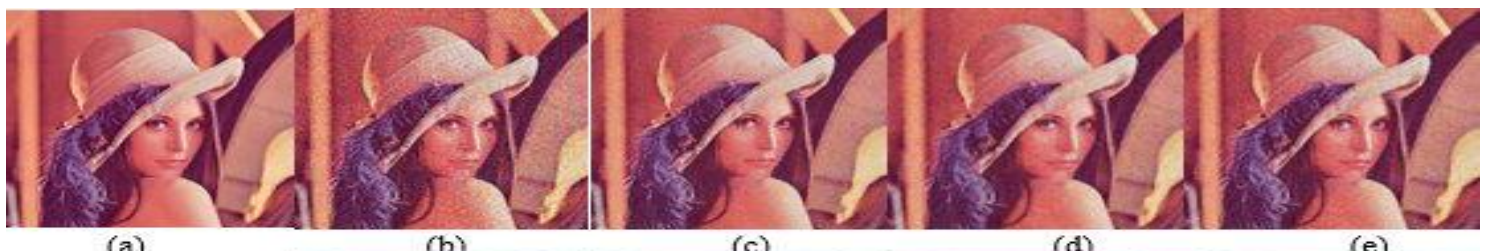

(a) (b) (c) (d) (e)

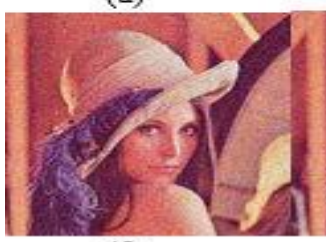

(f)

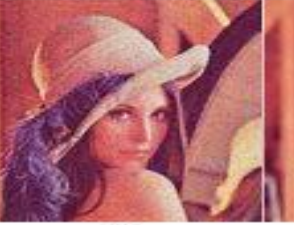

(g)

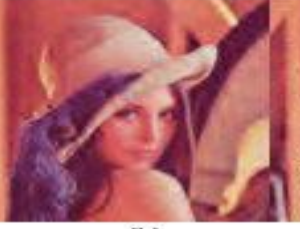

(h)

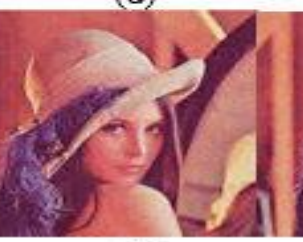

(i)

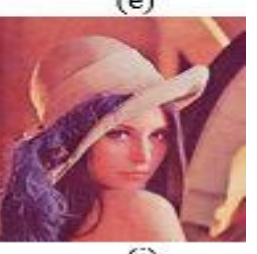

(i)

Fig. 17 a) Original Color Image b)Single Noisy Image (mean 0, variance 0.03) c) NLM d) BILF e) Gaussian f)ADF g) Guided h) Visu-Shrink SURE(Soft threshold) i) Visu-Shrink SURE(Hard threshold) j)DnCNN

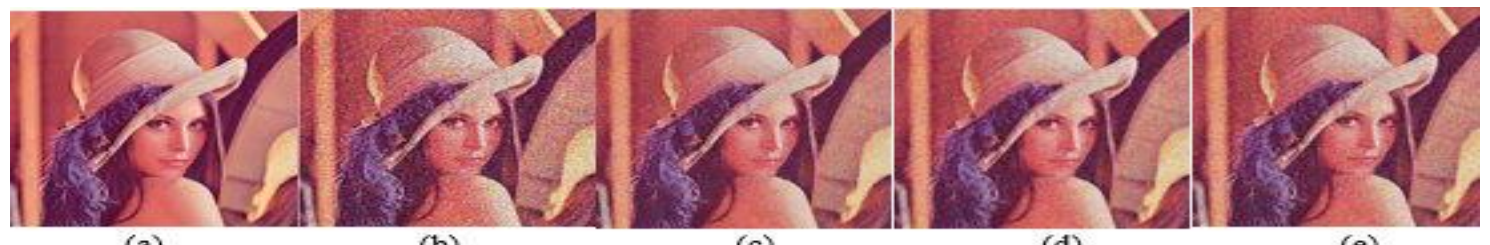

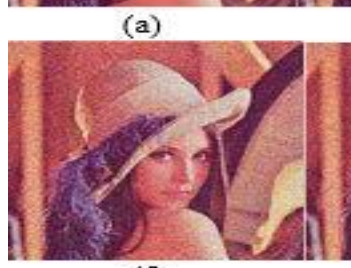

(f) (b)

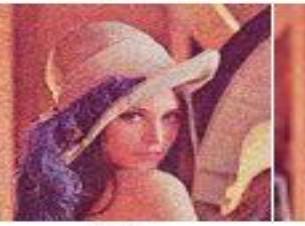

(g) (c)

(d) (e)

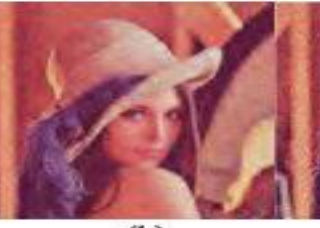

(h)

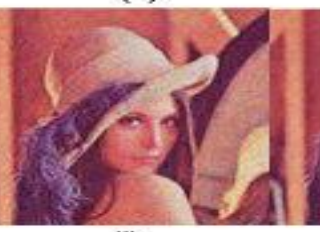

(i)

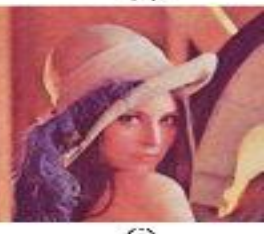

(i)

Fig. 18 a) Original Color Image b)Single Noisy Image (mean 0, variance 0.05) c) NLM d) BILF e) Gaussian f)ADF g) Guided h) Visu-Shrink SURE(Soft threshold) i) Visu-Shrink SURE(Hard threshold) j)DnCNN

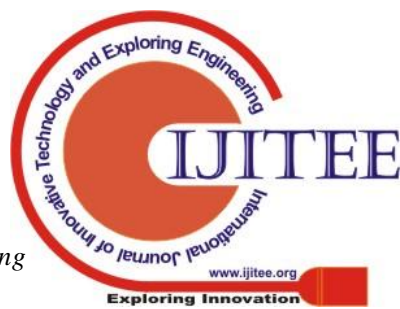




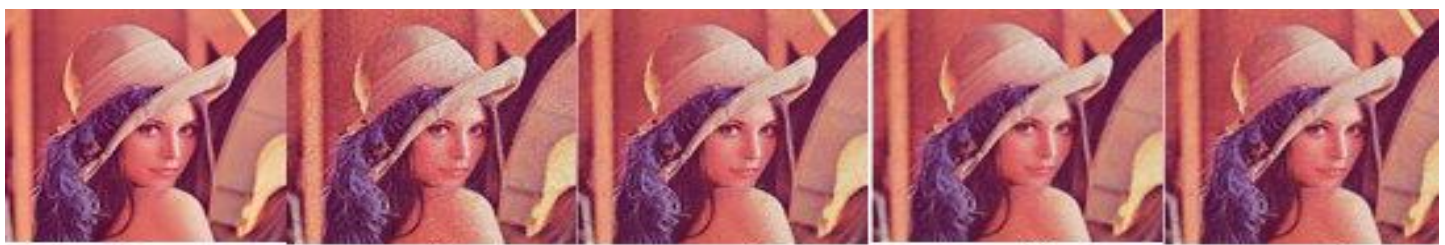

(a)

(b)

(c)

(d)

(e)

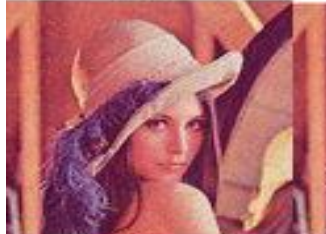

(f)

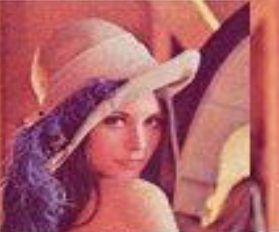

(g)

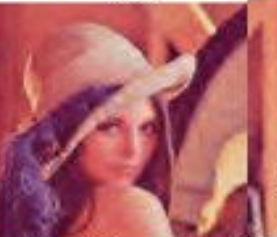

(h)

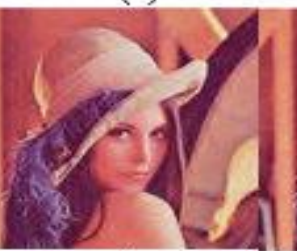

(i)

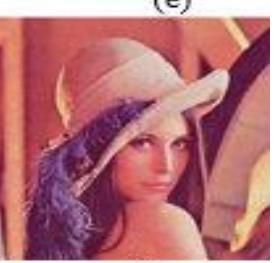

(j)

Fig. 19 a) Original Color Image b)Mixed Noisy Image (mean 0, variance 0.01) c) NLM d) BILF e) Gaussian f)ADF g) Guided h) Visu-Shrink SURE(Soft threshold) i) Visu-Shrink SURE(Hard threshold) j)DnCNN

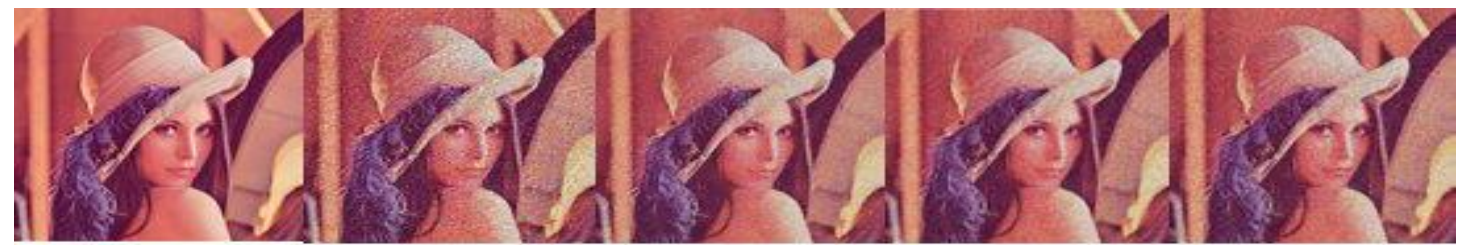

(a)

(b)

(c)

(d)

(e)

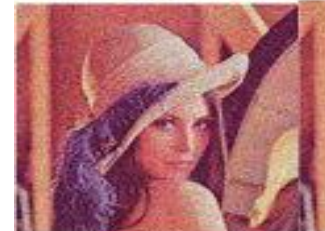

(f)

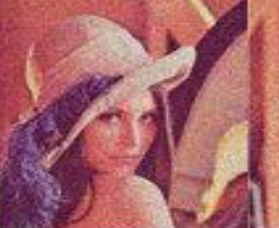

(g)

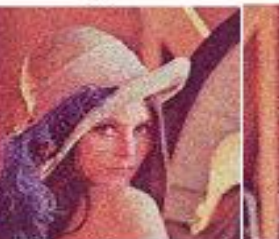

(h)

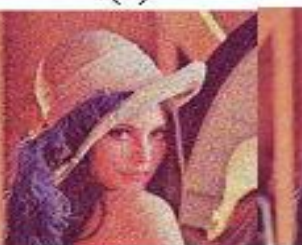

(i)

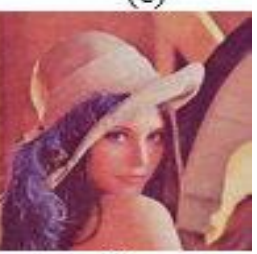

(j)

Fig. 20 a) Original Color Image b)Mixed Noisy Image (mean 0, variance 0.03) c) NLM d) BILF e) Gaussian f)ADF g) Guided h) Visu-Shrink SURE(Soft threshold) i) Visu-Shrink SURE(Hard threshold) j)DnCNN

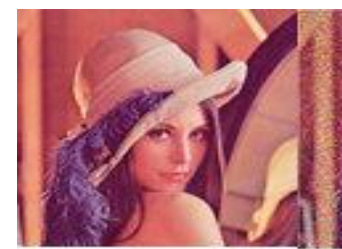

(a)

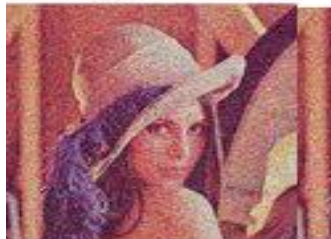

(f)

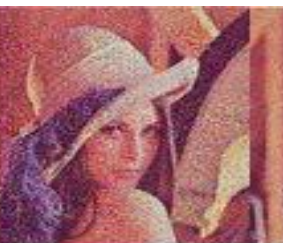

(b)

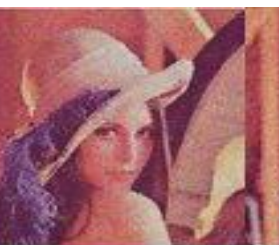

(c)

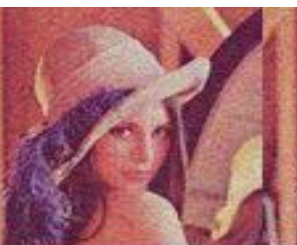

(d)

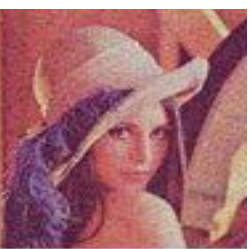

(e)

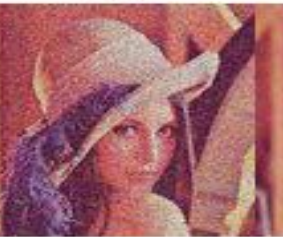

(g)

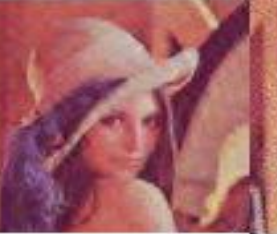

(h)

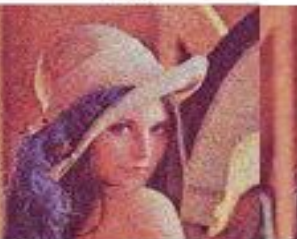

(i)

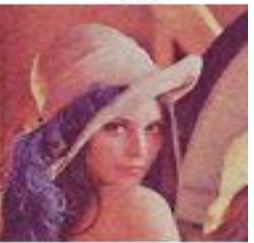

(i)

Fig. 21 a) Original Color Image b)Mixed Noisy Image (mean 0, variance 0.05) c) NLM d) BILF e) Gaussian f)ADF g) Guided h) Visu-Shrink SURE(Soft threshold) i) Visu-Shrink SURE(Hard threshold) j)DnCNN

\section{CONCLUSION:}

A short review of various noise models and de-noising filters followed by the comparative analysis of various de-noising filters are presented in this paper. This comparative study is performed on both the grayscale and color images. And also, the analysis is performed with single noise and mixed noise. From the study, it is evident that DnCNN outperforms the standard filters that are used for de-noising the images. The limitations of those standard filters are also presented here. The filters from the spatial domain, Transform domain and neural network approach are taken into account for comparative analysis. Among these DnCNN is found to be the best because it detects noise and artifacts of other high-frequency images. This DnCNN can be easily implemented along with any neural network-based approaches.

\section{REFERENCES:}

1. [1] R. C. Gonzalez, Richard e. woods, Digital image processing 2 (2002) 550570. 
2. H. Guo, J. E. Odegard, M. Lang, R. A. Gopinath, I. W. Selesnick, C. S. Bur-rus, Wavelet based speckle reduction with application to sar based atd/r, in: Proceedings of 1 st International Conference on Image Processing, volume 1, IEEE, 1994, pp. 75-79.

3. R. D. Nowak, Wavelet-based rician noise removal for magnetic resonance imag-ing, Image Processing 8 (1999) 1408-1419.

4. E. W. Weisstein, Rayleigh distribution, sigma 13 (2004) 14.

5. C. Pal, A. Chakrabarti, R. Ghosh, A brief survey of recent edgepreserv-ing smoothing algorithms on digital images, arXiv preprint arXiv:1503.07297 (2015).

6. P. Jain, V. Tyagi, A survey of edge-preserving image denoising methods, In-formation Systems Frontiers 18 (2016) 159-170.

7. P. Jain, V. Tyagi, Spatial and frequency domain lters for restoration of noisy images, IETE Journal of Education 54 (2013) 108-116.

8. I. Pitas, A. N. Venetsanopoulos, Nonlinear digital lters: principles and appli-cations, volume 84, Springer Science Business Media, 2013.

9. D. Yang, J. Sun, Bm3d-net: A convolutional neural network for transform-domain collaborative ltering, IEEE Signal Processing Letters 25 (2017) 55-59.

10. L. Liu, C. P. Chen, Y. Zhou, X. You, A new weighted mean lter with a two-phase detector for removing impulse noise, Information Sciences 315 (2015) 1-16.

11. C. Kandemir, C. Kalyoncu, O. Toygar, A weighted mean lter with spatial-bias elimination for impulse noise removal, Digital Signal Processing 46 (2015) 164-174.

12. D. Marr, E. Hildreth, Theory of edge detection, Proceedings of the Royal So-ciety of London. Series B. Biological Sciences 207 (1980) 187-217.

13. V. Chaudhary, V. Kumar, Fusion of multi-exposure images using recursive and gaussian lter, Multidimensional Systems and Signal Processing (2019) 1-16.

14. G. Deng, L. Cahill, An adaptive gaussian lter for noise reduction and edge detection, in: 1993 IEEE Conference Record Nuclear Science Symposium and Medical Imaging Conference, IEEE, 1993, pp. 1615-1619.

15. A. Makandar, B. Halalli, Breast cancer image enhancement using median lter and clahe, International Journal of Scienti c Engineering Research 6 (2015).

16. J. Zhang, An e cient median lter based method for removing randomvalued impulse noise, Digital Signal Processing 20 (2010) $1010\{1018$.

17. J. Chen, X. Kang, Y. Liu, Z. J. Wang, Median ltering forensics based on convo-lutional neural networks, IEEE Signal Processing Letters 22 (2015) 1849-1853.

18. P. Perona, J. Malik, Scale-space and edge detection using anisotropic di u-sion, IEEE Transactions on pattern analysis and machine intelligence 12 (1990) 629-639.

19. J. Xu, Y. Jia, Z. Shi, K. Pang, An improved anisotropic di usion lter with semi-adaptive threshold for edge preservation, Signal Processing 119 (2016) 80-91.

20. S. Tebini, H. Seddik, E. B. Braiek, An advanced and adaptive mathematical function for an e cient anisotropic image ltering, Computers Mathematics with Applications 72 (2016) 1369-1385.

21. C. Tomasi, R. Manduchi, Bilateral ltering for gray and color images., in: Iccv, volume 98, 1998.

22. J. Zhang, G. Lin, L. Wu, C. Wang, Y. Cheng, Wavelet and fast bilateral lter based de-speckling method for medical Control 18 (2015) 1-10.

23. K. V. Thakur, O. H. Damodare, A. M. Sapkal, Poisson noise reducing bilateral lter, Procedia Computer Science 79 (2016) 861-865.

24. A. Buades, B. Coll, J.-M. Morel, A non-local algorithm for image denoising, in: 2005 IEEE Computer Society ultrasound images, Biomedical Signal Processing and

Conference on Computer Vision and Pattern Recognition (CVPR'05), volume 2, IEEE, 2005, pp. 60-65.

25. P. Sudeep, P. Palanisamy, J. Rajan, H. Baradaran, L. Saba, A. Gupta, J. S. Suri, Speckle reduction in medical ultrasound images using an unbiased non-local means method, Biomedical Signal Processing and Control 28 (2016) $1-8$

26. G. Wang, Y. Liu, W. Xiong, Y. Li, An improved non-local means lter for color image denoising, Optik 173 (2018) 157-173.

27. D. L. Donoho, J. M. Johnstone, Ideal spatial adaptation by wavelet shrinkage, biometrika 81 (1994) 425-455.

28. T. Blu, F. Luisier, The sure-let approach to image denoising, IEEE Transac-tions on Image Processing 16 (2007) 2778-2786.

29. X. Zhang, The sure-let approach using hybrid thresholding function for image denoising, Computers Electrical Engineering 70 (2018) 334-348.

30. X. Zhang, Improved interscale-based sure-let approach to image denoising, Pro-cedia Computer Science 107 (2017) 819-823.

31. S. G. Chang, B. Yu, M. Vetterli, Adaptive wavelet thresholding for image denoising and compression, IEEE transactions on image processing 9 (2000) 1532-1546.

32. A. Achim, P. Tsakalides, A. Bezerianos, Sar image denoising via bayesian wavelet shrinkage based on heavytailed modeling, IEEE Transactions on Geo-science and Remote Sensing 41 (2003) 1773-1784.

33. I. Z. Nishu, M. F. Tanjim, A. Badrudduza, M. Al Mamun, A new image de-speckling method by srad lter and wavelet transform using bayesian threshold, in: 2019 International Conference on Electrical, Computer and Communication Engineering (ECCE), IEEE, 2019, pp. 1-6.

34. Y. Zhao, Y. Li, X. Dong, B. Yang, Low-frequency noise suppression method based on improved dncnn in desert seismic data, IEEE Geoscience and Remote Sensing Letters (2018).

35. K. Zhang, W. Zuo, Y. Chen, D. Meng, L. Zhang, Beyond a gaussian denoiser: Residual learning of deep cnn for image denoising, IEEE Transactions on Image Processing 26 (2017) 3142-3155

36. H. R. Shahdoosti, Z. Rahemi, Edge-preserving image denoising using a deep convolutional neural network, Signal Processing 159 (2019) 20

\section{Authors Biography:}

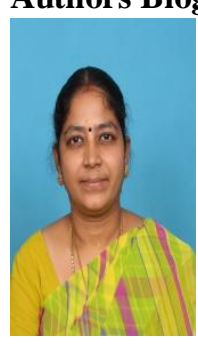

Dr Suganya Devi $K$ is an Assistant Professor in the Department of Computer Science and Engineering, National Institute of Technology (N.I.T) Silchar. She did her Ph.D. at Anna University Chennai, India. She completed her M.E. in Multimedia Technology (Gold Medallist) at Anna University, Chennai in 2008. Her area of research includes Image Processing, Video Segmentation, Cloud computing and Security. She has published more than 20 research papers in various reputed International journals with high Impact factor.

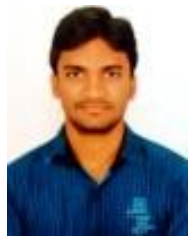

Satish Kumar satti is a Research scholar in Department of CSE at NIT silchar. He completed his M.Tech in CSE and B.Tech in Information Technology. He has 6 years' experience in teaching and his area 


\section{AN EFFICIENT NOISE SEPARATION TECHNIQUE FOR REMOVAL OF GAUSSIAN AND MIXED NOISES IN \\ MONOCHROME AND COLOR IMAGES}

of interests are Image Processing, Bio medical Image Processing, Machine Learning, and Deep Learning.

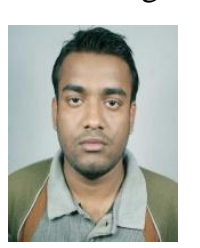

Prasenjitdhar is a Research schalar in Department of CSE at NIT silchar. He completed his M.Tech in CSE at Tripura University and B.Tech in CSE at Tripura Institute of technology.His area of interests are Bio medical image Processing, Machine Learning, and Deep Learning.

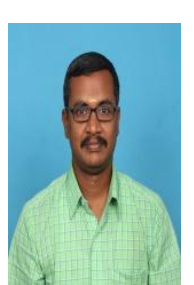

Dr P. Srinivasan is an Assistant Professor in the Department of Physics, National Institute of Technology (N.I.T) Silchar. He did his Ph.D. at Anna University Chennai, India and PostDoctoral Research in University of Kerala, India. His area of research includes nonlinear optics and Modelling in Crystal Growth He has published more than 50 research papers in various reputed International journals with high Impact factor. 